great success in these difficult cases is to transilluminate the tissues with a powerful cold-light source such as the long flexible fibreoptic light used for cystoscopy, bronchoscopy, etc, which is usually readily available from theatres or intensive therapy units. By placing the light source in the palm of the patient's hand vessels on the dorsum can be clearly seen, where none have been previously visible, by inspecting the skin surface. A similar technique can be used for the foot. The wrist can also be transilluminated allowing easy access to the radial artery for cannulation and removing arterial blood to measure blood gases.

C G Peters

Anaesthetic Department, Sheffield Children's Hospital,
Sheffield S10 2 TH

\section{Intravenous urography}

SIR,-Drs Benvon Cramer and Gerald de Lacey (6 September, p 661) discuss special circumstances in which the standard procedure for intravenous urography should be altered. One important modification that they do not stress is the value of taking delayed films up to 24 hours after the administration of contrast medium, if there is delay to the excretion of contrast by one, or both kidneys. This may give useful anatomical information as to the level of obstruction and may indicate the cause of that obstruction. It is particularly helpful in the management of renal and ureteric colic, and in cases of renal failure thought to be due to ureteric obstruction.

The delay in excretion gives a crude estimate of the degree of obstruction, but I would agree this is more accurately and better assessed by radioisotope methods. Intravenous urography, however, is readily available 24 hours a day in most hospitals and continues to be the first-line investigation for patients presenting as emergencies with obstruction to the upper urinary tract. Bradford Royal Infirmary,
West Yorkshire BD9 6RJ

\section{Dietary fibre and calcium excretion in diabetes}

SIR,-We read with interest the report by $\mathrm{Mr}$ P J R Shah and others (9 August, p 426) on the beneficial effect of bran in hypercalciuria. Our experience of the use of guar gum in maturityonset diabetes supports their findings and suggests that a similar effect on calcium excretion may be achieved with various components of dietary fibre.

We studied the long-term effects of dietary supplementation with guar gum ( $21 \mathrm{~g}$ a day) taken in three divided doses at the main meals, in a double-blind cross-over comparison with placebo in nine insulin-independent diabetics. The main interest was in the effects on glucose and lipid metabolism, and the results will be published elsewhere. The serum and urinary calcium levels were monitored during the experiment, and it was found that after three months of guar gum supplementation the urinary excretion of calcium was significantly less $(2.64 \pm 0.67 \mathrm{mmol} / 24 \mathrm{~h}(106 \pm 27 \mathrm{mg} /$ $24 \mathrm{~h}$ ), mean $\pm \mathrm{SE}$ ) than during the control period $(3.80 \pm 0.74 \mathrm{mmol} / 24 \mathrm{~h}(152 \pm 30 \mathrm{mg} /$ $24 \mathrm{~h}), \mathrm{p}<0.025)$. No difference was observed in mean serum calcium concentration between the two periods. There was a concomitant reduction in mean glucosuria by about $50 \%$ during the guar gum period.

The observed reduction in calcium excretion is partially, but probably not entirely, explained by the reduced degree of glucosuria, because a positive correlation has been found between glucosuria and calcium excretion in diabetes. The rather marked reduction in calcium excretion, however, which was evident in eight out of the nine diabetics studied, probably also reflects a reduction in calcium absorption due to the administration of guar gum. The possible mechanism by which guar gum could reduce calcium absorption is obscure, since in contrast to the phytate- and uronic acidcontaining components of fibre, guar gum does not bind calcium in vitro. ${ }^{2}$

The study should be repeated and the results confirmed in non-diabetics before any general conclusions on the effects of suar gum on calcium excretion can be drawn. Our findings -although inconclusive-are in support, however of the results of Shah and co-workers and, in addition, suggest that different analogues of dietary fibre may be of practical importance in absorptive hypercalciuria.

ANTti Aro MATTI UUSITUPA TIMO KORHONEN ONNI SIITONEN

Department of Medicine, University of Kuopio, SF-70210 Kuopio 21, Finland

1 McNair P, Madsbad S, Christensen MS, et al. Acta Endocrinol (Copenh) 1979;90:463-72. 2 James WPT, Branch WJ, Southgate DAT. Lancet
1978;i:638-9.

\section{Managing extrapyramidal disorders}

SIR,-The issue of 6 September, (p 655 and p 690) contains references to the management of two extrapyramidal disorders, but the advice proffered in each case requires clarification.

Minerva quotes* a study from Boston $^{1}$ in which drug holidays were employed in patients with Parkinson's disease in an attempt to restore therapeutic benefit. While the success of this manoeuvre is open to question, the effect of stopping levodopa treatment can be quite dramatic. Next day the patient will have reverted to his usual Parkinsonian state, and a few days later he may deteriorate to become more immobile than ever before. Such patients may become mute, bedridden, and unable to swallow. Clearly this procedure is not to be undertaken lightly and is best carried out under supervision in hospital.

In response to a question concerning tests that might predict whether a child of a patient with Huntington's disease will develop the illness, your anonymous expert states that: "in descendants of families who have not yet developed the choreaform movements the use of levodopa at about $3 \mathrm{~g}$ a day will produce chorea in those who are at the moment asymptomatic." Certainly this is true in about a third of those at risk, but it is not yet known for certain whether such individuals will inevitably go on to develop Huntington's disease. In any case, the wisdom of undertaking such a predictive test is very questionable. There is no guarantee that the disease may not be precipitated earlier by doing so, and the psychological impact of provoking chorea in such an individual at risk can be disastrous; it is like passing a death sentence, a burden many cannot carry. Until effective treatment for this disease is available, I, and many others concerned in the management of this disease, would proscribe the routine use of predictive tests. Elsewhere ${ }^{2} I$ have argued that in the present state of therapeutic impotence it is reasonable to ask those at risk who are lucky enough not to carry the gene for the disease to share the burden of uncertainty with those who have inherited it, so as to provide the latter with some hope.

\section{D MARSDEN}

University Department of Neurology,

Institute of Psychiatry,

London SE5 8AF

1 Direnfeld LK, Feldman RG, Alexander MP, KellyHayes M. Neurology 1980;30:785-8.

2 Barette J, Marsden CD. Psychol Med 1979;9:327-36.

$*_{*}^{*}$ We regret the page number quoted by Minerva was wrong.-ED, $B M F$.

\section{Acupuncture and postherpetic neuralgia}

SIR,-I was interested to read of the experience of Dr G T Lewith and Dr Jennifer Field (30 August, $p$ 622) in the use of acupuncture for postherpetic neuralgia.

I use acupuncture in the pain relief clinic at Ipswich but have not found it very helpful in postherpetic neuralgia. It does seem logical to try to prevent this distressing complication, and there is some evidence that sympathetic nerve block given as early as possible in the acute attack of herpes zoster may diminish the incidence of neuralgia. ${ }^{1}$ I have begun to use sympathetic or somatic nerve block in the acute phase, and I find that the pain is decreased and the attack seems to be reduced in length. In the few cases treated none have progressed to postherpetic neuralgia, but further experience is needed to evaluate this approach.

\section{The Ipswich Hospital,
Suffolk 1PI 3PY}

Clive Jolly

${ }^{1}$ Colding A. Der Anaesthesist 1967;16:172-4.

\section{Percutaneous central venous cannulation}

SIR,-The hazards of subclavian cannulation have again been rightly emphasised by $\mathrm{Mr}$ J L Peters and others (30 August, $p$ 618). The suggested use of ultrasound to identify the subclavian artery and vein may be worthy of trial although we doubt if it will, in practice, reduce the incidence of pneumothorax. This is the major hazard of subclavian puncture particularly in inexperienced hands. A simpler alternative may be puncture of the internal jugular vein at the level of the cricoid. If the Vygon silicone catheter with detachable hub is used it is a simple matter to make a skin tunnel from the site of puncture down to the chest wall below the clavicle using the introducing cannula. This was originally described by Dr Powell-Tuck for the subclavian vein. ${ }^{1}$ Puncture of the internal jugular vein is virtually free of the risk of pneumothorax and the use of the skin tunnel should prevent the problem of infection in patients' necklines. The major hazard is puncture of the carotid artery, but we suspect that this is less dangerous for the patient than tension pneumothorax occurring in the intensive therapy unit or on the operating table following subclavian puncture. We have now used this technique in a small number of patients-usually those on 